\title{
Sensitivity of X-ray Burst Models to Uncertainties in Nuclear Processes
}

\author{
Karl Smith ${ }^{\star 1,2,3}$, A. Matthew Amthor ${ }^{1,2,3}$, Richard Cyburt ${ }^{1,2,3}$, Ryan Ferguson ${ }^{1,2,3}$, \\ Alexander Heger ${ }^{3,4,5}$, Emily Johnson ${ }^{2}$, Matthew Klein ${ }^{1,2,3}$, Zachary Meisel ${ }^{1,2,3}$, \\ Thomas Rauscher $^{6}$, Alexander Sakharuk ${ }^{1,2,7}$, Friedrich-K. Tielemann ${ }^{6}$, Hendrik \\ Schatz $^{1,2,3}$, Scott Warren ${ }^{8}$, Michael Wiescher ${ }^{1,9}$ \\ ${ }^{I}$ National Superconducting Cyclotron Laboratory (NSCL) \\ ${ }^{2}$ Dept. of Physics and Astronomy, Michigan State University \\ ${ }^{3}$ The Joint Institute for Nuclear Astrophysics (JINA) \\ ${ }^{4}$ Los Alamos National Laboratory \\ ${ }^{5}$ Dept. of Physics and Astronomy, University of Minnesota \\ ${ }^{6}$ Dept. für Physik und Astronomie, Universität Basel \\ ${ }^{7}$ Florida Gulf Coast University \\ ${ }^{8}$ Dept. of Electrical and Computer Engineering, Michigan State University \\ ${ }^{9}$ Dept. of Physics, University of Notre Dame \\ E-mail: ksmithenscl.msu.edu
}

\begin{abstract}
The underlying nuclear reaction sequence in Type I X-ray bursts is the rp-process. We examine the sensitivity of current X-ray burst models to uncertainties in nuclear reaction rates in terms of predicted X-ray light curves and final produced ashes. Many of the relevant reaction rates have significantly large uncertainties that impact the results of X-ray burst models. We used an updated nuclear reaction network and ran almost 400,000 simulations with a single-zone X-ray burst model to determine the influence of individual reaction rate variations. We also explored the validity of single-zone approximations as tools to investigate nuclear physics by comparing to a full 1-D multi-zone model. We show that a properly calibrated single-zone model can save computation time and give similar results to multi-zone models. Using the calibrated singlezone model we identify the most significant reaction in the X-ray burst.
\end{abstract}

10th Symposium on Nuclei in the Cosmos

Mackinac Island, Michigan, USA

27 July - 1 August, 200

\footnotetext{
* Speaker

$\dagger$ This work is supported by the National Science Foundation under grants PHY-06-06007 (NSCL) and PHY-0216783 (JINA)
} 


\section{Introduction}

Multi-zone X-ray burst models simulate thermonuclear explosions on the surface of accreting neutron stars in binary systems [1]. X-ray bursts are observed frequently by $\mathrm{X}$-ray observatories and are affected strongly by nuclear reactions [2]. We examine the sensitivity of $\mathrm{X}$-ray burst models to uncertainties in nuclear reaction rates through the rapid proton capture process, known as the rp-process as well as the ap-process. This is done best by using a full 1-D multi-zone model to simulate an X-ray burst. Other studies including one by A. Parikh et al. [3] presented at this conference used post-processing techniques to address the same problem of identifying the most sensitive reactions. We have used models that evolve both the temperature and density as the burst progresses. This method is more physical than using a predetermined profile. We used the multi-zone hydrodynamics code KePLER. [4]. The multi-zone model divides the upper layers of the star into zones which have independent isotopic abundances. This allows the model to simulate burning processes and energy transport in full 1-D.

In addition extended sequences of bursts are calculated where ashes from earlier bursts to affect later ones, this is known as compositional "inertia" [5]. Full 1-D models require a large amount of computing time. Therefore it is not possible to examine variations of all possible reactions. We therefore use a single-zone model of Schatz et al. [6] to first identify the most sensitive reactions and then use the multi-zone model to confirm their sensitivity. We have found that the final composition and light curve can be well approximated with a single-zone model if the ignition conditions are chosen properly.

We have used the REACLIB V1.0 [7] set of reaction rates as the baseline for comparison. At this conference Meisel et al. [8] presented more details about the updated reaction rate library. The single-zone model contains a reaction network of over 650 nuclei ensuring that the burst is not truncated.

\section{Single-Zone Model Calibration}

The single-zone model is calibrated to the full 1-D multi-zone model by adjusting ignition conditions until the light curve and final ashes matched between the two models as well as possible. The light curve and final composition are used as signatures of the X-ray bursts. The burst is chosen from the chain of bursts in the multi-zone model. The first burst that most closely approximated the median lightcurve of the full burst chain, disregarding early bursts which tend to be outliers, is used as the calibration burst. Using this selection process we chose the third burst in the chain of bursts as the burst in which we would calibrate the single-zone against. We chose an initial composition from a zone in the multi-zone model that underwent hydrogen and helium burning during the third burst. The composition is taken at a time just before the third burst of a baseline multi-zone model when the ${ }^{15} \mathrm{O}$ abundance had peaked, ensuring the burst had not yet occurred. We concluded that the best initial conditions to use for the single-zone model was the initial composition from this particular zone in the hydrogen burning layer at an initial temperature of $0.386 \mathrm{GK}$ and pressure of $1.73 * 10^{22} \mathrm{erg} / \mathrm{cm}^{3}$. 

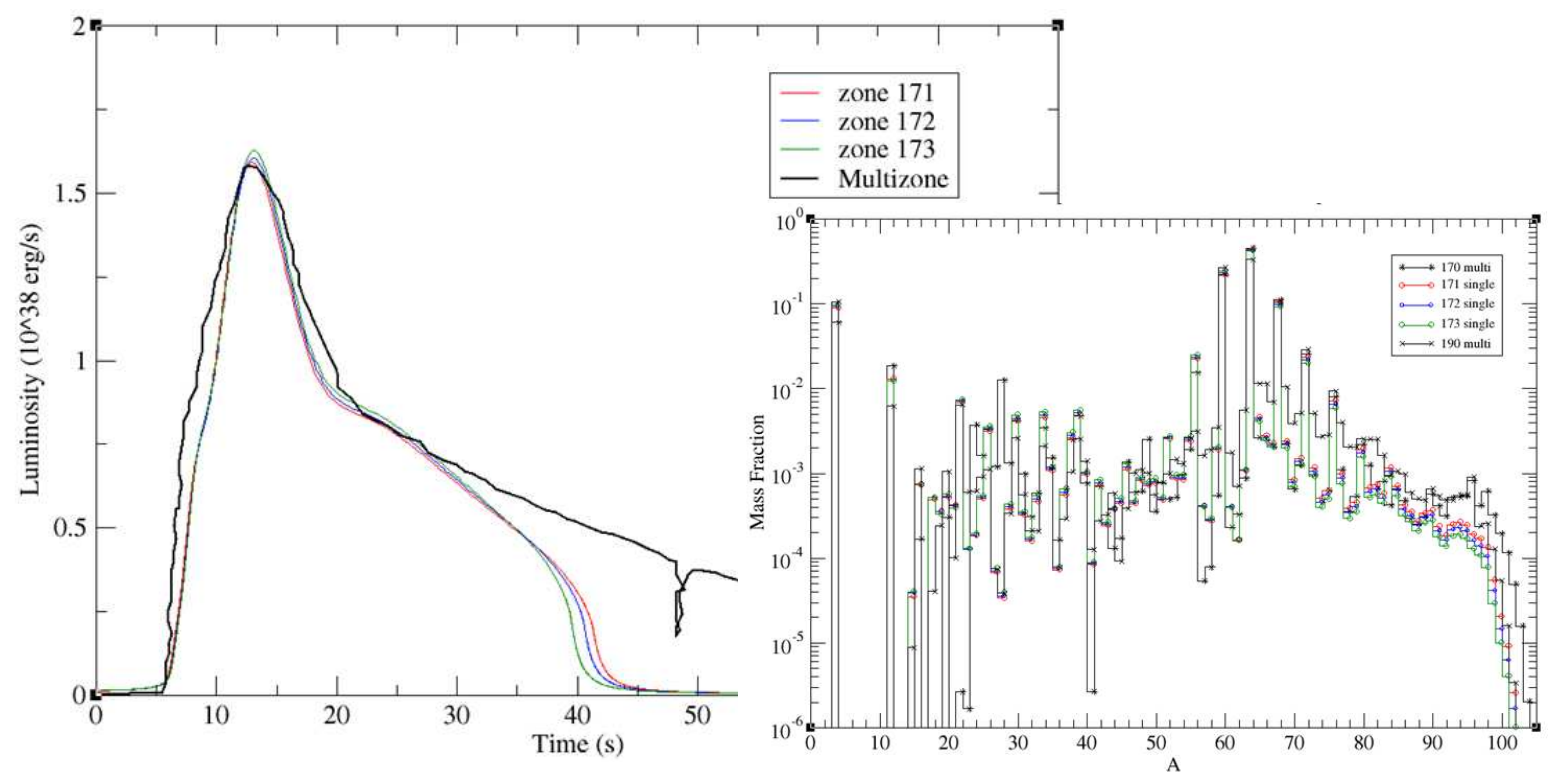

FIGURE 1: Estimated luminosity from one-zone model compared to a median lightcurve from the multi-zone model as a function of time for the three differing compositions. Inset shows composition of ashes versus mass number. The dip in the multi-zone model is a numerical defect caused when a cold layer is accreted onto the surface of the star.

As shown in Figure 1, the single-zone model matches the multi-zone model quite well. The rise and peak are similar as well as the initial section of the tail. There are some deviations between the models, for example, the single-zone model shows the final section of the tail decaying more rapidly compared to the multi-zone model, this is not unexpected as the singlezone model does not include the details of radiation transport. Also, the single-zone model produces final ashes that match in composition to the multi-zone model reasonably well at the depth where the hydrogen is consumed during a burst.

\section{Identifying Reactions}

We ran over 800,000 simulations with the calibrated single-zone model, varying reaction rates by a factor of 100 up and down, to identify the reaction rates the X-ray burst is most sensitive to. The list of reaction rates is composed of roughly $850(\mathrm{p}, \gamma)$ rates and $850(\mathrm{p}, \alpha)$ rates. We adjusted a single reaction rate at a time and also varied two reactions simultaneously.

Light curves and final ashes are examined for each run performed and compared to the nominal burst, which used the REACLIBV1.0 rate library [7]. We are particularly interested in light curve shape and rise time, as these are important observables. Constraining these allows for a comparison of models to observed X-ray bursts. Deviations in the final mass fraction are also important, final composition can show production of heavier isotopes or changes in relative abundances. Varying some rates may cause the burst to process more lighter material into heavier material. Often there are correlations between changes in light curve and final composition. A longer burst will process more light isotopes to heavy isotopes and burn more hydrogen fuel. We will see this situation as longer burst duration on the light curve and higher abundances in the final composition. The reaction rate changes are rated by the amount of change in the simulated light curve, and those with significant impact have their realistic 
uncertainties estimated. These new limits are then used in the multi-zone model to evaluate that model's sensitivity to the realistic uncertainties.

Figure 1 shows the most significant impacts. Changes in the ${ }^{59} \mathrm{Cu}(\mathrm{p}, \gamma)$ rate causes the largest change with an increase in peak luminosity and a much faster decay about $20 \mathrm{sec}$ after the peak. Changes in a reaction rate with lighter reactants will cause a deviation earlier in the burst. Changing the ${ }^{27} \mathrm{P}(\mathrm{p}, \gamma)$ rate, we see the light curve begins to deviate almost immediately in the rise of the burst with a similar peak and slightly longer decay. The heavier reactants do not begin to deviate until later towards the park luminosity. Many of the reactions are also identified by Parikh et al. [3], but others, for example ${ }^{27} \mathrm{P}(\mathrm{p}, \gamma)$ and ${ }^{63} \mathrm{Ga}(\mathrm{p}, \gamma)$, are not identified by Parikh.

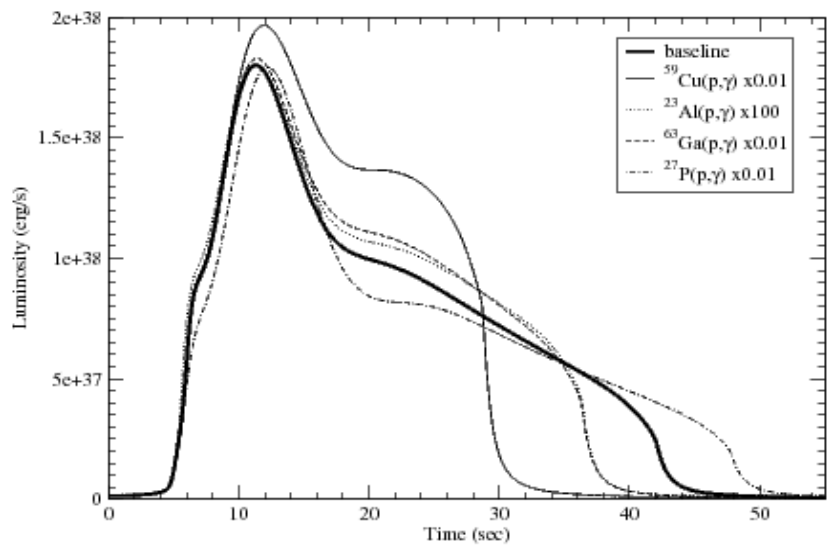

FIGURE 2: Estimated luminosity from one-zone model as a function of time for the four most sensitive reaction rates identified.

We also examined composition of final ashes as another measure of burst change used in this study. Figure 3 shows that the composition of the ashes is also substantially affected by varying reaction rates, such an impact was also shown by Parikh et al. [3]. All three variation plotted modify at least one isobar by an order of magnitude and many others by several factors. We see that for the burst with the ${ }^{59} \mathrm{Cu}(\mathrm{p}, \gamma)$ reaction decreased, production of lighter isotopes is increased and production of heavier isotopes is decreased. This large effect can be explained by the $\mathrm{NiCu}$ I cycle below and matches with the shorter X-ray burst duration as depicted above.

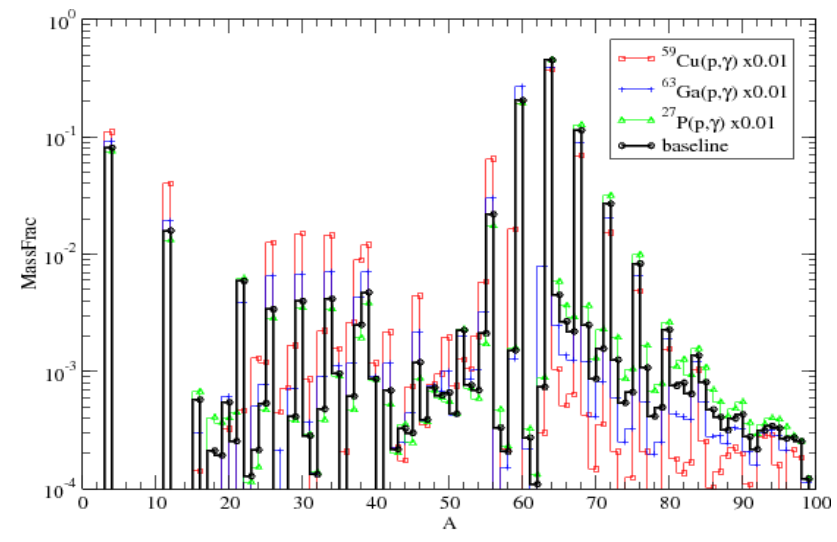

FIGURE 3: Estimated composition from final ashes of one-zone model as a function of mass number for the three most sensitive reaction rates identified. Mass fractions are summed along isobaric chains 
Reducing the ${ }^{27} \mathrm{P}(\mathrm{p}, \gamma)$ rate, reduces the production of lighter isotopes and increases for heavier isotopes. This matches with a longer burst duration and shows that deviations in light curve and final composition are correlated. A change in light curve is usually accompanied by a change in the final ashes.

Cycle formation can occur when a $(\mathrm{p}, \gamma)$ rate and a $(\mathrm{p}, \alpha)$ rate compete, this is seen in the $\mathrm{NiCu}$ I cycle has been described by Van Wormer et al. [9]. This explains the large sensitivty to the decreased ${ }^{59} \mathrm{Cu}(\mathrm{p}, \gamma)$ reaction rate, the $(\mathrm{p}, \alpha)$ branch is not faster and feeds back to ${ }^{56} \mathrm{Ni}$ which then proton captures and beta decays back to ${ }^{59} \mathrm{Cu}$, a much smaller fraction of material is processed through the proton capture toward heavier isotopes.

\section{Conclusions}

We have found that the behavior of a multi-zone model can be approximated using a single-zone model when choosing the correct ignition conditions. Using this calibrated model we identify the list of reactions to which the single-zone model is most sensitive. These reactions are currently being run with the multi-zone model and sensitivities examined.

The identified reactions are now being run with more realistic uncertainties in both the single and multi-zone models. This will show how reaction rate measurements can best reduce uncertainty in X-ray burst models.

\section{Acknowledgements}

This work is supported by the National Science Foundation under grants PHY-06-06007 (NSCL) and PHY-02-16783 (JINA)

\section{References}

[1] Amthor, A.M. et al, Sensitivity of Type I X-Ray Bursts to rp-Process Reaction Rates, Proc. Science, PoS(NIC-IX) 068 (2006).

[2] Schatz, H. et al, X-ray Binaries, Nuclear Physics A 477 (2006) 601

[3] A. Parikh et al, The Effects of Variations in Nuclear Processes on Type I X-Ray Burst Nucleosynthesis, astro-ph/0802.2819 (2008)

[4] S. E. Woosley et al, Models for Type I X-Ray Bursts with Improved Nuclear Physics, Astrophysical Journal Supplement Series 151 (2004) 75.

[5] Taam, R. X-ray Bursts from Thermonuclear Runaways on Accreting Neutron Stars, Astrophysical Journal 241 (1980) 358.

[6] H. Schatz et al, End Point of the rp Process on Accreting Neutron Stars, Physical Review Letters 86 (2001) 3471 .

[7] http://www.nscl.msu.edu/ nero/db

[8] Z. Meisel et al, The rp-process in Type-I X-ray Bursts with REACLIB V1.0, PoS(NIC-X) (2008)

[9] Van Wormer et al, Reaction rates and reaction sequences in the rp-process 1994, ApJ, 432, 326.

[10] Schatz, H. The rp process and x-ray bursts, Proc. Science PoS(NIC-IX) 002 (2006) 